\title{
Review: Detection and Identification of Unknown Substances
}

\author{
Supapan Seraphin
}

Department of Materials Science and Engineering, University of Arizona, Tucson, AZ 85721

The questions - How can you identify an unknown substance? and When might you come into contact with unknown chemicals in the real world? are listed on a website introducing various analytical chemistry techniques such as chromatography, spectroscopy, crystallography and mass spectrometry [1]. The questions became more relevant to our daily life recently due to the world events of terrorisms and natural disasters (e.g. hurricanes and tsunamis).

In 1999, the Centers for Disease Control and Prevention (CDC) established the Laboratory Response Network (LRN) [2]. The LRN's purpose is to run a national network of 140 labs that can respond quickly to acts of chemical or biological terrorism, emerging infectious diseases, and other public health threats and emergencies. It includes federal, state and local public health, environmental, food testing, veterinary, military, and international labs in Canada, UK and Australia. LRN labs are designated as either national, reference, or sentinel. Designation depends on the types of tests a laboratory can perform and how it handles infectious agents to protect workers and the public. National labs have unique resources to handle highly infectious agents and the ability to identify specific agent strains. Reference labs (confirmatory reference) can perform tests to detect and confirm the presence of a threat agent. These labs ensure a timely local response in the event of a terrorist incident. Sentinel labs represent the thousands of hospital-based labs that are on the front lines and have direct contact with patients. They could be the first facility to spot a suspicious specimen and are responsible to refer to the right reference lab. The LRN has been put to the test on several occasions. In 2001, a Florida LRN reference laboratory discovered the presence of Bacillus anthracis in a clinical specimen. $B$. anthracis causes anthrax. LRN labs tested 125,000 samples by the time the investigation was completed. This amounted to more than 1 million separate tests. LRN BioWatch labs test air filters for threat agents. Tests include polymerase chain reaction (PCR), which can quickly detect the presence of an agent's unique DNA. The LRN also developed tests and materials needed to identify the unique DNA sequence of the virus that causes severe acute respiratory syndrome (SARS).

The Agency for Toxic Substances and Disease Registry (ATSDR) in CDC, issues general information on identification techniques, located in Managing Hazardous Materials Incidents Volumes I and II [3]. The Unidentified Chemical protocol provides basic victim management recommendations but the techniques for a specific chemical could provide information which would allow more effective patient treatment.

In terms of devices, scientists in various laboratories are successful in making devices or sensors that can detect harmful substances from safe distance and quickly in the case of emergency. Scientists at the U.S. Department of Energy's Brookhaven National Laboratory have been awarded U.S. patent for a portable device that can detect and identify unknown chemical and biological substances from safe distances of 2 to 50 meters [4]. The device, called a mini-Raman light detection and ranging (lidar) sensor, uses light scattering principle to identify the substance. The process takes only minutes. The system is small enough to be transported and carried by emergency responders. 
Against the backdrop of the war on terrorism, researchers at Sandia National Laboratories are perfecting two versions of a compact, handheld chemical detection device (Fig. 1) that acts as a small chemical laboratory [5]. They could be taken into the field to help thwart threats from terrorists. Another device developed at Sandia called Sentinel II, is used to screen not only bags but also the travelers (Fig. 2). The devices use Ion Mobility Spectrometry, which performs a real-time chemical analysis and essentially identifies a "fingerprint" of a target substance. Even if a person has only a minute concentration of explosives, drug residue, or chemical agent on his or her skin or clothing, the substance will be detected. The technology efficiently traps the target particles and vapors from a large volume of air, then directs the concentrated chemical sample to detector for analysis, which makes possible the detection of very low concentrations of explosive chemicals.

By combining three technologies into a single system - an ion trap mass spectrometer for analysis, a high-powered microscope for viewing, and a laser for ionizing samples - the Forensic Science Center has created something entirely new for forensic analysis: imaging laser-ablation mass spectroscopy (Fig. 3) [6]. Conceived in 1994 and still being refined, this new process allows considerably more accuracy in analyzing samples than standard mass spectroscopy.

The presentation will review the state-of-the-art devices and principles of detecting and identifying harmful substances that can potentially be a treat to the public.

\section{Reference:}

[1] http://misterguch.brinkster.net/identify.html

[2] http://www.bt.cdc.gov/lrn/factsheet.asp

[3] http://www.atsdr.cdc.gov/mhmi.html

[4] www.bnl.gov/bnlweb/pubaf/pr/PR display.asp

[5] www.sandia.gov/media/NewsRel/NR2002/portallaunch.htm

[6] Forensic Science Center, Science \& Tech. Review, August 1995

[7] Assistance from Tula Jutarosaga, Jovanny Diaz, Shane Smith, Leifee Li, and Ahmed Alobeidi is gratefully acknowledged.


Fig. 1 Handheld chemical detection device

Fig. 2 Sentinel II walk-through portal can screen seven passengers per minute for residues of explosives and other chemicals

Fig. 3 Imaging laser-ablation mass spectroscopy 